Methods We conducted detailed literature searches of MEDLINE, EMBASE, PsycInfo and the Cochrane library, searched trial registration sites, contacted authors if results have not been published and hand searched reference lists. Three categories of search terms were used; paediatric, CFS/ME and recovery.

Inclusion criteria Randomised controlled trials or observational studies of participants $<19$ years old with a diagnosis of CFS/ $\mathrm{ME}$, related to a Western Health Care system, some measure of recovery (partial or full) reported and the time taken to reach it. Results 21 papers were identified. The study populations ranged from 1 to 64 participants, their duration of illness ranged from 3 months to 7 years between studies and also showed great variety within each study. Some studies used a single measurement outcome for recovery, others measured several and some integrated multiple outcomes to formulate one value for recovery. The recovery rate ranged from $25-100 \%$ in those accessing treatment and $4.5-100 \%$ in those without specialist care. School attendance was the most common measurement outcome $(\mathrm{n}=$ 11 ), of which 4 of these combined this with at least one other measure. 7 studies measured physical ability as an outcome, 8 used fatigue and 9 measured a global improvement that was either self-rated or qualitatively assessed by an investigator. 2 studies described recovery as no longer fulfilling the diagnostic criteria.

Conclusion Recovery rates are relatively high in children accessing specialist treatment however, the discrepancies between the measurement outcomes, makes interpretation of recovery rates difficult.

\section{G359 THE DIAGNOSIS, MANAGEMENT AND OUTCOMES OF PAEDIATRIC EOSINOPHILIC OESOPHAGITIS: A FIVE-YEAR RETROSPECTIVE ANALYSIS OF THE WEST OF SCOTLAND POPULATION}

${ }^{1} \mathrm{M}$ Goddard, 'DM Flynn, ${ }^{2} \mathrm{D}$ Penman, ${ }^{1} \mathrm{AR}$ Barclay, ${ }^{1} \mathrm{H}$ Duncan, ${ }^{1} \mathrm{RK}$ Russell, ${ }^{1} \mathrm{R}$ Hansen. ${ }^{1}$ Paediatric Gastroenterology, Yorkhill Hospital, Glasgow, UK; ${ }^{2}$ Paediatric and Perinatal Pathology, Southern General Hospital, Glasgow, UK

\subsection{6/archdischild-2015-308599.315}

Background Eosinophilic oesophagitis (EO) is a recently described allergic condition characterised by oesophageal dysmotility with progression to stricture formation in severe cases. Diagnosis is histological, based on $>15$ per high-powered field in oesophageal biopsies. Treatment options remain limited to dietary modification (including elemental feeds), proton pump inhibitors (PPI) and topical/ systemic steroids. There is sparse population data on the condition.

To report demographic data on children with EO, establish the current burden of disease and review effectiveness of treatments with clinical and histopathological outcomes.

Methods All patients diagnosed with EO between 2008-2013 in RHSC Glasgow were retrospectively identified by joint review of all oesophageal histopathology reports $(n=1060)$ with a Consultant Paediatric Pathologist (DP) and findings correlated with clinical symptoms, treatment and outcomes. Data was analysed using Microsoft Excel 2007 and SPSS version 22.0.

Results Within the West of Scotland 30 children (mean age 9.1, range 0.7-15.2) were diagnosed with EO between January 2008 and May 2013 with no obvious increase in annual incidence. $22(73 \%)$ were male. The most common presenting symptoms were abdominal pain (43\%), vomiting (37\%) and dysphagia (33\%), with food bolus obstruction in 27\%. Most (77\%) had concurrent atopic disease. At diagnosis the mean number of oesophageal eosinophils per high-powered field was 61, ascertained with a mean of 5 biopsies from 3 different oesophageal regions. The mean peripheral eosinophil count at diagnosis was 1.8 (range 0.0-27.0).

PPIs were clinically effective in 19/21 (70\%) patients and 14/ 16 showed histological improvement. All 17 patients receiving dietary therapy demonstrated clinical improvement and 14/15 showed histological improvement; 11 (40\%) had topical steroids, clinically effective in $10 / 11$ patients with $7 / 9$ showing histological improvement. However, 4 (13\%) progressed to oral steroids. There was considerable overlap between treatments. At one year follow-up 93\% were clinically improved and 86\% showed endoscopic improvement.

Conclusions The total numbers of patients diagnosed was surprisingly small. Whilst treatment with PPI is effective, most patients also required dietary or steroid therapy. A significant number required oral steroids. A departmental guideline is being developed on the basis of these results and further work is required to establish frequency of repeat endoscopy.

\section{G360 THE EPIDEMIOLOGY AND OUTCOME OF BILIARY ATRESIA IN SCOTLAND 2002-2013}

${ }^{1} E$ Sutton, ${ }^{2} \mathrm{P}$ Henderson, ${ }^{3} \mathrm{R}$ Tayler, ${ }^{2} \mathrm{~K}$ Fraser, ${ }^{2} \mathrm{~J}$ Cowieson, ${ }^{4} \mathrm{D}$ Goudie, ${ }^{5} \mathrm{~K}$ McIntyre, ${ }^{5} \mathrm{D}$ Kastner-Cole, ${ }^{6} \mathrm{C}$ Cameron, ${ }^{6} \mathrm{~S}$ Loganathan, ${ }^{7} \mathrm{P}$ Rogers, ${ }^{7} \mathrm{D}$ Mitchell, ${ }^{8} \mathrm{C}$ Delahunty, ${ }^{2} A R$ Barclay, ${ }^{2} R$ Hansen. 'University of Glasgow, Glasgow, UK; ${ }^{2}$ Paediatric Gastroenterology, Yorkhill Hospital, Glasgow, UK; ${ }^{3}$ Paediatric Hepatology, Leeds Children's Hospital, Leeds, UK; ${ }^{4}$ Paediatrics, Raigmore Hospital, Inverness, UK; ${ }^{5}$ Paediatrics, Ninewells Hospital, Dundee, UK; ${ }^{6}$ Paediatric Gastroenterology, Royal Aberdeen Children's Hospital, Aberdeen, UK; 'Paediatric Gastroenterology, Royal Hospital for Sick Children, Edinburgh, UK; ${ }^{8}$ Paediatrics, Wishaw General Hospital, Wishaw, UK

\subsection{6/archdischild-2015-308599.316}

Background Biliary atresia (BA) is a rare, poorly understood liver disease of infancy that is fatal if not treated through early biliary drainage via the Kasai procedure. BA surgery was rationalised to three UK centres in 2002 following data supporting improved outcomes in institutions performing $>5$ Kasai per year. We have previously shown that outcomes in Scottish children were worse than expected in the years following initial rationalisation.

Aims We aimed to expand the post-rationalisation cohort of BA cases in Scotland to examine epidemiology and outcomes.

Methods Outcomes of the previously published 2002-2009 incident cohort was first expanded. New Scottish incident cases of BA born between 2010-2013, were obtained using data from specialist nurse/team knowledge. New data collection focussed on demographics, details of Kasai and outcomes (particularly 2 year transplant-free survival [2YTFS]). Accurate regional and national population data was obtained from the General Register Office for Scotland and statistics performed in $\mathrm{R}$ with Poisson regression analysis for incidence trends.

Results 48 infants were initially identified, of whom 5 were excluded from outcome analysis (three with Kasai performed in Edinburgh, one with $<1$ yr follow-up post-Kasai and one born outside Scotland). Three infants required immediate liver transplantation; 43 infants underwent Kasai. Median age at Kasai in the full cohort was 55 days (range 19-96) and showed significant improvement from 61 days in 2002-2009 to 49 days in 2010-2013 ( $\mathrm{p}<0.0001$ ). Of those with available data, $45 \%$ cleared their jaundice (bilirubin $<20$ umol/l) six months post-Kasai; 2YTFS was $40 \%$. BA incidence in Scotland was $0.68 / 10,000$ live births and appeared stable $(0.66 / 10,000$ in 
2002-2009, 0.71/10,000 in 2010-2013). A cluster of cases was identified in Lanarkshire where the incidence was $1.26 / 10,000$ live births, significantly higher than the rest of Scotland $(0.59$ / 10,000, $\mathrm{p}=0.033)$.

Conclusion BA atresia incidence appears relatively stable in Scotland but with an unexplained cluster of cases in Lanarkshire; examination of this cluster may provide epidemiological insight into disease pathogenesis Despite a significant reduction in time to Kasai, the 2YTFS in Scotland remains disappointing, is lower than the pre-rationalisation figure of $65 \%$ and is not currently an endorsement of centralisation of BA surgery in Scotland.

\section{G361 PARENTAL PHOTOGRAPHIC MONITORING OF ASCITES}

C MacDougall, L Saych, A Brightwell, K Blair, P Clarke, G Briars. Paediatrics, Norfolk and Norwich University Hospital, Norwich, UK

\subsection{6/archdischild-2015-308599.317}

Aims Primary Chylous Ascites is a rare cause of abdominal distension in infants. Our patient had six weeks of intravenous octreotide and total parenteral nutrition followed by medium chain triglyceride formula feeding. We describe his monitoring by girth measurement and parental photography.

Methods A four week old boy who presented with worsening abdominal distension and discomfort had ultrasound confirmed ascites. At paracentesis, $100 \mathrm{ml}$ of chylous fluid was aspirated. His clinical course was monitored by serial girth measurement and parental photography using a modern mobile telephone.

Results Variability in abdominal circumference measurements (Figure 1) made detection of clinical improvement more difficult than it was with serial photography (Figure 2). His mother found the photographs helpful in monitoring her son's progress. Conclusion This superiority of parental photography over formalised girth measurement monitoring in our patient mandates critical review of these techniques. Informal parental photography has been used in assessment of dermatological conditions ${ }^{1}$ and inguinal hernias. ${ }^{2}$ Inter-observer variability in measurements of abdominal circumference is recognised. ${ }^{3}$ Parental photography may increase parents' perceived involvement in their child's care in such chronic treatment and promote a family centred approach. We speculate that measurements from technical photographs ${ }^{4}$ as employed in sports biomechanical studies ${ }^{5}$ will be the most sensitive tool for clinical assessment of ascites.

\section{REFERENCES}

1 Nayler JR. Clinical photography: a guide for the clinician. I Postgrad Med. 2003:49:256-262

2 Kawaguchi AL. Inguinal hernias can be accurately diagnosed using the parent's digital photographs when the physical examination is nondiagnostic. J Pediatr surg. 2009;44:2327-2329

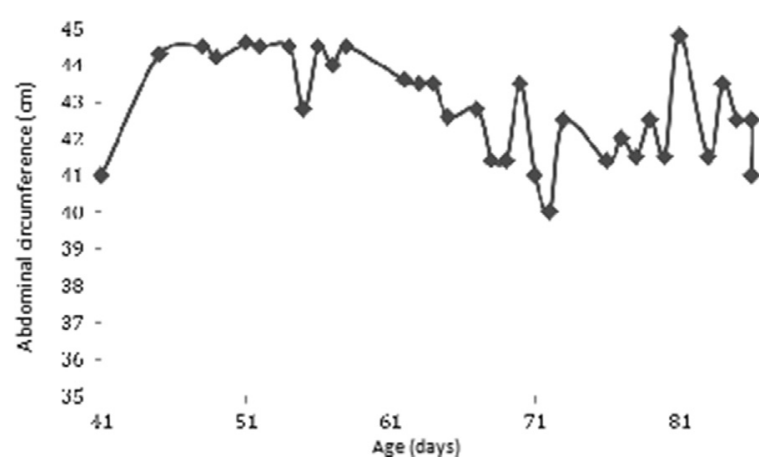

Abstract G361 Figure 1 Abdominal circumference measurements

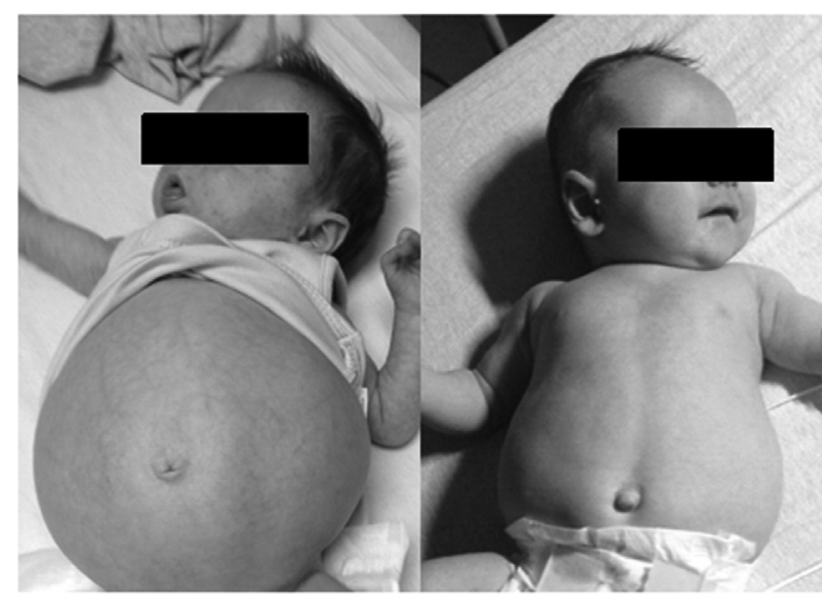

Abstract G361 Figure 2 Clinical improvement via photography

3 Johnson TS, Engstrom JL, Gelhar DK. Intra and interexaminer reliability of anthropometric measurements of term infants. I Pediatr Gastroenterol Nutr. 1997;24:497-505

4 Jensen RK. Estimation of the biomechanical properties of three body types using a photogrammetric method. J Biomech. 1978;11:349-358

5 Wicke J, Lopers B. Validation of the volume function with Jensen's (1978) elliptical cylinder model. J App/ Biomech. 2003;19:3-12

\section{G362(P) CHILD NUTRITION: A “NEED TO FEED” FUTURE DOCTORS}

${ }^{1} \mathrm{~S}$ Bali, ${ }^{2} \mathrm{H}$ Jacob, ${ }^{2} \mathrm{C}$ Fertleman. ${ }^{1}$ Paediatric Accident and Emergency, Chelsea and Westminster Hospital, London, UK; ${ }^{2}$ Department of Paediatrics, The Whittington Hospital, London, UK

\subsection{6/archdischild-2015-308599.318}

Aims Parents may ask doctors working in a range of specialties for advice on childhood nutrition. The Department of Health states that all doctors working with children should engage in health promotion. Ensuring the provision of high quality information may help prevent obesity and optimise the health of children and young people.

As many doctors may have limited postgraduate exposure to paediatrics, medical school is an ideal time to learn about the importance of childhood nutrition. We decided to explore the knowledge, skills and attitudes of medical students about providing guidance on childhood nutrition.

Methods We sent a survey to all medical students undertaking their paediatric placement at one teaching hospital between 2012-13. We asked the students whether they felt comfortable advising about breastfeeding, infant and toddler nutrition, obesity, food allergies and infant weighing schedules. We also enquired from them about maternal alcohol consumption during breastfeeding and about a crying baby whose mother was struggling to breastfeed.

We collected data using software from SurveyMonkey ${ }^{\mathrm{TM}}$ and analysed it using Microsoft Excel $^{\mathrm{TM}}$.

Results 82 students were asked to fill in the survey. 47(57\%) responded. 32(68\%) felt uncomfortable advising about breastfeeding and toddler nutrition. Despite this, 33(70\%) felt they would advise a mother struggling with breastfeeding rather than referring her to the health visitor. $32(68 \%)$ and $26(55 \%)$ thought that with help they could provide information about obesity and food allergies respectively. Regarding maternal alcohol consumption whilst breastfeeding 27(57\%) knew the appropriate advice about drinking minimally or in moderation. 